\title{
A case of intracranial migration and rapid spontaneous resolution of traumatic acute subdural hematoma
}

\author{
Melih Yuksel $^{1 *}$, Caner Saglam², Mehmet Cagri Goktekin², Mahmut Tas ${ }^{2}$, Abdullah Mesut $^{3}$
}

Abstract

Traumatic acute subdural hematoma (ASDH) is one of the most destructive forms of traumatic brain injury (TBI), involving estimated mortality rates of 40-60\%. Traumatic ASDH is a frequently seen life-threatening condition requiring emergency intervention. Spontaneous resolution and migration of ASDH are both rare entities, the causes of which are still not fully understood. The few existing cases in the literature of both rapid spontaneous resolution of SDH and of subdural migration are generally in the form of case reports. Publications concerning migration of SDH mainly involve migration to the lumbar region. We encountered no previous reports of intracranial migration of ASDH accompanied by rapid spontaneous resolution. This report describes a case of intracranial migration and spontaneous resolution within $24 \mathrm{~h}$ in a 61 -year-old male patient with traumatic acute subdural hematoma (ASDH), together with a discussion of the relevant mechanisms.

Key words: intracranial subdural hematoma, emergency medicine, traumatic brain injury

Introduction

Traumatic acute subdural hematoma (ASDH) is one of the most destructive forms of traumatic brain injury (TBI), involving estimated mortality rates of $40-60 \%$ (1). Hematoma developing post-traumatically generally results in tearing of the bridging veins between the medial facet of the cerebral hemisphere, the falx cerebri, the superior sagittal sinus and the parieto-occipital cortex (2). Monitoring and treatment of ASDH is based on serial computerized tomography (CT) of the brain, depending on the patient's neurological status and thickness of hematoma. Generally, surgical intervention is recommended in subdural hematomas (SDHs) greater than $10 \mathrm{~mm}$, while surgery is not effective in hematomas smaller than $3 \mathrm{~mm}$. Debate still continues regarding whether surgical intervention or a conservative approach is preferable in patients with a thickness of 5-10 $\mathrm{mm}$ and a Glasgow Coma Scale (GCS) score of 9-11 (3).

Rapid resolution of intracranial ASDH was first reported in $1986(4,5)$. The time to resolution of ASDH in the literature varies between the first few hours after trauma to a few days $(3,6)$.

Migration of acute hematoma in the subdural area is not a clinical rarity. There are few reports of migration of SDH in the literature, however, and those there are generally involve migration to the spinal canal (7-11). The purpose of this report was to describe a case of intracranial migration in posttraumatic acute subdural hematoma, followed by spontaneous resolution
Case

A 61-year-old male was brought to the emergency department after a fall of approximately 5 meters. Vital findings on arrival were TA: 120/70 mmhg, pulse: $88 / \mathrm{min}$, respiration rate: $20 / \mathrm{min}$ and oxygen saturation: 94 . GCS score was 15 . At physical examination, ecchymosis was determined in the right maxillary and frontotemporal region, laceration and crepitation in the right wrist, and edema, deformity and crepitation in the central third of the right femoral region. Sensitivity was determined in thoracic vertebrae 9-12.

Imaging techniques revealed open partial fracture at the distal end of the right radius, shaft fracture of the right femur, fissure-type fracture in the T9, 11 and 12 corpus vertebrae not extending to the spinal canal and plastering SDH in the right frontotemporal region (Figure 1).

Laboratory findings were WBC: 12.58 , HGB: 12.3, PLT: 161,000 and INR: 1.22. The patient was admitted to the intensive care unit. Control tomography of the brain performed 6 hours later revealed migration of the $\mathrm{SDH}$ in the right frontotemporal region to the right parieto-occipital region (Figure 2). Tomography of the brain performed after $24 \mathrm{~h}$ revealed no finding of intracranial haemorrhage. The patient had no neurological deficit. Cranial and cervical magnetic resonance imaging (MRI) was performed in order to determine complete resolution and presence or absence of migration to the spinal canal. 
Cranial MRI revealed no findings of haemorrhage and cervical MRI revealed no findings of migration (Figure 3). The patient had no neurological deficit due to the extremity fractures and was transferred to the orthopaedic clinic.

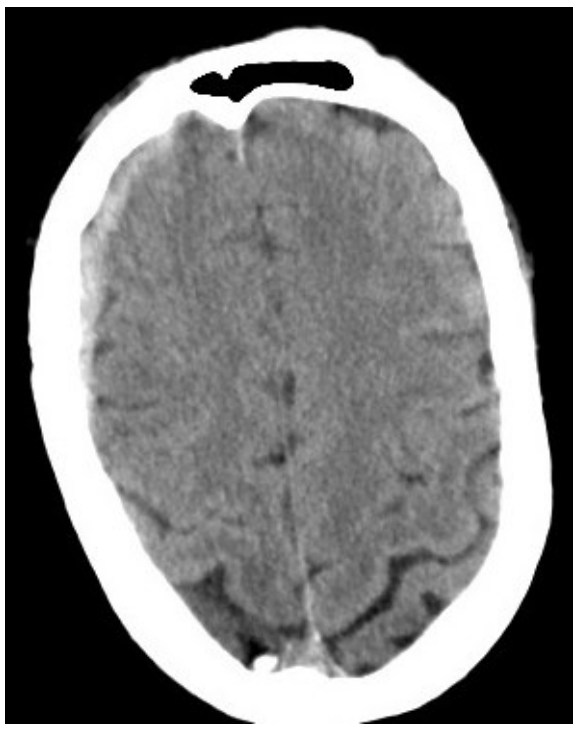

Figure 1. Subdural hemorrhage in the right frontotemporal region

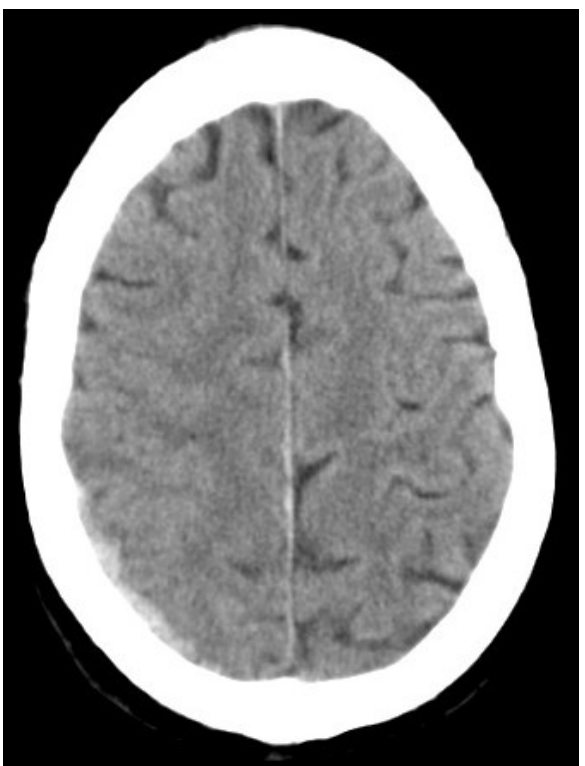

Figure 2. Subdural hemorrhage in the right parieto-occipital region
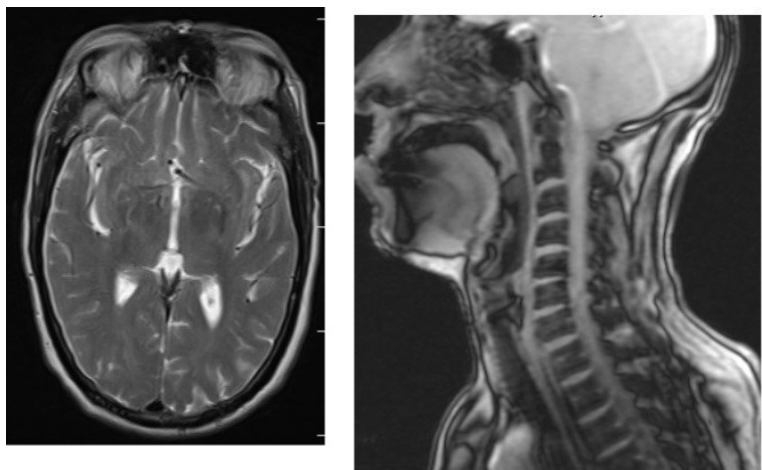

Figure 3. Cranial MRI and cervical MRI
Discussion

Traumatic ASDH is a neurological emergency constituting $10-20 \%$ of all major head traumas (2). The few existing cases in the literature of both rapid spontaneous resolution of $\mathrm{SDH}$ and of subdural migration are generally in the form of case reports. Publications concerning migration of SDH mainly involve migration to the lumbar region. A disposition to hemorrhage, blunt trauma, anticoagulant or antiaggregant therapy and invasive procedures such as lumbar puncture and epidural or spinal anesthesia all play a role in the etiology of migration to the spinal canal. It may also, albeit rarely, occur spontaneously (10).

Lumbar SDH was first described in 1948 by Schiller et al., in a 16-month-old male patient (12). Migration of intracranial SDH to the spinal canal was first described by Bortolotti et al. They reported a case of traumatic subdural bleeding in a 23-year-old woman with no spinal trauma findings (7).

Most cases of migrating SDH in the literature involve spontaneous $\mathrm{SDH}$, although traumatic cases have also been reported. Yang et al. reported a case of spontaneous subdural and spinal hematoma as a result of lower back ache and paraparesis developing after 1 week in a 35-year-old woman presenting with symptoms of headache and dizziness. No vascular abnormality (arteriovenous fistula or vascular malformation) was determined at cerebral or spinal angiography in that case (8).

Gilad et al. described a case of migration to the spinal canal of SDH resulting from spontaneous anterior communicating artery aneurysm rupture without trauma in a 47-year-old man with a history of hypertension, with headache and lower back pain for the previous 3 days and with no subarachnoid hemorrhage (9).

Moscovici et al. reported migration to the spinal canal of SDH resulting from minor head trauma in an 88-year-old male patient (10). Similarly, Li et al. reported migration to the spinal canal of cranial SDH developing following trauma in a 26-year-old man (11).

Kapsalaki et al. published a series of four cases of spontaneous resolution and redistribution of ASDH. These cases of traumatic SDH consisted of 2 male and 2 female patients requiring no surgical intervention. Duration of spontaneous resolution varied between 6.5 $\mathrm{h}$ and 7 days. Coagulation disorder was determined in only one case. That case (INR $>2.8$ ) had GCS of 8 and SDH thickness of $18 \mathrm{~mm}$. In the other 3 cases, GCS was 7-8 and SDH thickness 8-9 mm. (3). Yadav et al. reported spontaneous resolution after $72 \mathrm{~h}$ in a 55year-old male patient with GCS 6 with traumatic SDH and subarachnoid hemorrhage (SAH) (6).

The causes of spontaneous resolution of SDH are not fully established. There are various theories on the subject. According to one, rapid resolution of ASDH results from redistribution of blood, and it has therefore been suggested that redistributed blood that 
cannot be visualized at CT can be seen at MRI (5) According to another hypothesis, a rise in intracranial pressure following cerebral edema may lead to obliteration of ASDH (13). Another theory regarding hematoma resolution is washing and drainage of the hematoma by cerebrospinal fluid (CSF) thanks to tearing of the arachnoid membrane during trauma $(14,15)$.

Kundra et al. described spontaneous resolution and extracranial redistribution of ASDH and stated that ASDH gave rise to scalp hematoma by passing through a dural tear or calvarial fracture with direct pressure on the soft tissue, thus exhibiting redistribution (16). Another similar study suggested that linear skull fractures assisted distribution of ASDG into the extracranial area, as a result of which SDH progresses toward scalp hematoma through the bone fracture and meningeal tear pathway (17). There was no bone fracture in our case.

Another study showed that the presence of another SDH on the opposite side of the same hemisphere caused one hematoma to shrink while the other expanded (18). Cohen et al. suggested that cerebral atrophy developing in association with HIV facilitated resolution of ASDH (19).

$\mathrm{Wu}$ et al. suggested that a hematoma volume of less than $30 \mathrm{ml}$, and location near the sylvan fissure and in the frontotemporal or temporoparietal region affected spontaneous resolution of ASDH (20). The resolution mechanism in our case is compatible with the theory proposed by $\mathrm{Wu}$ et al. because the ASDH in our case had a small volume and was located in the frontotemporal area.

\section{Conclusion}

Our scan of the literature revealed no previous reports of concomitant intracranial migration of ASDH and rapid intracranial resolution. We think that this interesting case will make a useful contribution to the literature.

\section{References}

1. Kalanithi P, Schubert RD, Lad SP, et al.Hospital costs, incidence, and inhospital mortality rates of traumatic subdural hematoma in the United States. J Neurosurg 2011;115(5):1013-18.

2. Tsui EYK, Ma KF, Cheung YK, et al.Rapid spontaneous resolution and redistribution of acute subdural hematoma in a patient with chronic alcoholism. Eur J Radiol 2000;36(1): 53-7.

3. Kapsalaki EZ, Machinis TG, Robison JS ,et al. Spontaneous resolution of acute cranial subdural hematomas. Clin Neurol Neurosurg 2007;109(3): 287-91.

4. Nagao $\mathrm{T}$, Aoki $\mathrm{N}$, Mizutani $\mathrm{H}$, et al.Acute subdura hematoma with rapid resolution in infancy: case report. Neurosurgery 1986;19(3):465-7.
Polman CH, Gijsbers CJ, Heimans JJ,et al. Rapid spontaneous resolution of an acute subdural hematoma. Neurosurgery 1986;19(3): 446-8.

Yadav YR , Agarwal M , Namdev H, et al.Rapid resolution of acute subdural hematoma : A case report and review of literature. Indian Journal of Neurotrauma 2011;8(1):45-8

7. Bortolotti C, Wang H, Fraser $\mathrm{K}$, et al.Subacute spinal subdural hematoma after spontaneous resolution of cranial subdural hematoma: causal relationship or coincidence? Case report. J Neurosurg 2004;100(4):372-4.

8. Yang MS, Tung YW, Yang TH, et al. Spontaneous spinal and intracranial subdural hematoma. J Formos Med Assoc 2009;108(3):258-61.

9. Gilad R, Fatterpekar GM, Johnson DM,et al. Migrating subdural hematoma without subarachnoid hemorrhage in the case of a patient with a ruptured aneurysm in the intrasellar anterior communicating artery. American Journal of Neuroradiology 2007;28(10): 2014-16.

10. Moscovici S, Paldor I, Ramirez de Noriega F, et al. Do cranial subdural hematomas migrate to the lumbar spine? J Clin Neurosci 2011;18(4):563-5.

11. Li CH, Yew AY, Lu DC. Migration of traumatic intracranial subdural hematoma to lumbar spine causing radiculopathy. Surgical Neurology International 2013;19(4): 81

12. Shiller F, Neligan G, Budtz-Olsen O. Surgery in hemophilia : A case of spinal subdural hematoma producting paraplegia. Lancet 1948;27(2): 842-5.

13. Makiyama Y, Katayama Y, Ueno Y, et al. Acute subdural hematomas spontaneously disappearing within 3 days following closed head injury. Nihon Univ J Med 1985;27: 123-7.

14. Fujioka S, Hamada J, Kaku M, et al. Rapid resolution of acute subdural hematoma. Report of two cases. Neurol Med Chir (Tokyo)1990;30(11): 827-31.

15. Niikawa S, Sugimoto S, Hattori T, et al.Rapid resolution of acute subdural hematoma. Report of four cases. Neurol Med Chir (Tokyo)1989;29(9): 820-4.

16. Kundra SN, Kundra R.Extracranial redistribution causing rapid spontaneous resolution of acute subdural hematoma. Neurol India 2005; 53(1): 124

17. Lou XH, Yang RJ. Spontaneous resolution of acute subdural hematoma in 3 cases. Chin J Traumatol 2000;16: 526

18. Kuroiwa $\mathrm{T}$, Tanabe $\mathrm{H}$, Takatsuka $\mathrm{H}$, et al. Rapid spontaneous resolution of acute extradural and subdural hematomas. J Neurosurg 1993;78(1): 126-8.

19. Cohen JE, Eger K, Montero A, et al. Rapid spontaneous resolution of acute subdural hematoma and HIV related cerebral atrophy: case report. Surg Neurol 1998;50(3):241-4.

20. Wu MC, Liu JX, Luo GC,et al. Rapid natural resolution of intracranial hematoma. Chin J Traumatol 2004;7(2):96100.

Copyright (C) 2014 The Author(s); This is an open-access article distributed under the terms of the Creative Commons Attribution License (http://creativecommons.org/licenses/by/4.0), which permits unrestricted use, distribution, and reproduction in any medium, provided the original work is properly cited. 